\title{
Do Pregnancy Outcomes of Women With False- Positive Early Gestational Diabetes Mellitus Differ From Those of Women With Normal Glucose Tolerance?
}

\section{Sayuri Nakanishi}

Yokohama City University Medical Center

Shigeru Aoki ( $\sim$ smyyaoki@yahoo.co.jp ) Yokohama City University Medical Center

Ryosuke Shindo

Yokohama City University Medical Center

Soichiro Obata

Yokohama City University Medical Center

Junko Kasai

Yokohama City University Medical Center

Etsuko Miyagi

Yokohama City University School of Medicine

\section{Research Article}

Keywords: Gestational diabetes mellitus, Early onset gestational diabetes mellitus, Pregnancy outcomes

Posted Date: December 21st, 2021

DOI: https://doi.org/10.21203/rs.3.rs-1180064/v1

License: (c) (i) This work is licensed under a Creative Commons Attribution 4.0 International License.

Read Full License 


\section{Abstract}

Background: To investigate whether false-positive early gestational diabetes mellitus (GDM) women can be managed similarly as normal glucose tolerance (NGT) women.

Methods: This retrospective study was conducted at a tertiary care center in Japan. Pregnancy and neonate outcomes of 117 singleton pregnancies with false-positive early GDM and 1774 singleton pregnancies with NGT who delivered after 22 weeks of gestation were compared. GDM was diagnosed according to the IADPSG criteria (patients having one or more of the following: fasting plasma glucose $\geq$ $92 \mathrm{mg} / \mathrm{dL}$ and a $75 \mathrm{~g}$ oral glucose tolerance test (OGTT) value $\geq 180 \mathrm{mg} / \mathrm{dL}$ at $1 \mathrm{~h}$, or $\geq 153 \mathrm{mg} / \mathrm{dL}$ at 2 h). Pregnant women diagnosed with GDM in early pregnancy who did not meet the diagnostic criteria on the second OGTT were defined as having false-positive early GDM. Women with false-positive early GDM did not receive any therapeutic intervention during gestation.

Results: Maternal age, pre-pregnancy BMI, and gestational weight gain were significantly higher in the false-positive GDM group than the NGT group. No significant differences were found in pregnancy outcomes, including gestational age, birth weight, large for gestational age rate, and cesarean delivery rate. Except for neonatal hypoglycemia rate, no significant differences were found in neonatal outcomes.

Conclusions: There were no clinically significant differences between early GDM false-positive women exhibiting GDM patterns only during early pregnancy and NGT women. False-positive early GDM women can be managed similarly as NGT women, suggesting that WHO diagnostic guidelines, applying the IADPSG criteria during early pregnancy, need revision.

\section{Background}

Since the diagnosis and treatment of gestational diabetes mellitus (GDM) improves pregnancy outcomes, $[1,2]$ screening for GDM in pregnancy is an important treatment practice. In 2010, the International Association of Diabetes and Pregnancy Study Groups (IADPSG) indicated that a diagnosis of GDM should be made if one or more of the following results are obtained from the $75 \mathrm{~g}$ oral glucose tolerance test (OGTT) conducted between 24 and 28 weeks of gestation: $92 \mathrm{mg} / \mathrm{dL}$ for a fasting plasma glucose (FPG) concentration, $180 \mathrm{mg} / \mathrm{dL}$ for a $1 \mathrm{~h}$ postprandial plasma glucose (1 h PG) concentration, or 153 $\mathrm{mg} / \mathrm{dL}$ for $2 \mathrm{~h}$ postprandial plasma glucose (2 h PG) concentration [3]. In 2010, physicians were also advised to make a diagnosis of GDM if an FPG of $92 \mathrm{mg} / \mathrm{dL}$ was observed in early pregnancy. In 2016, this recommendation concerning early pregnancy was retracted, and the IADPSG recommended that early-onset GDM be considered if an $\mathrm{HbA} 1 \mathrm{c}$ level of $5.9 \%$ or higher is observed in early pregnancy. [4] However, since 2013, the WHO has recommended that if any of the IADPSG threshold values are met at any time during pregnancy, GDM should be diagnosed. [5] In Japan, WHO diagnostic criteria for GDM have been employed since 2010. [6]

However, no conclusive evidence has yet been found suggesting that diagnosis and treatment of GDM in early pregnancy improves pregnancy outcomes. [7-9] Thus, to investigate the clinical significance of 
early-onset GDM, we conducted the Timing of Therapeutic Intervention for Gestational Diabetes Mellitus (TTIGDM) study, which was designed to evaluate the validity and efficacy of treating GDM (detected in early pregnancy) during mid-pregnancy. In the TTIGDM study, we monitored pregnant women diagnosed with GDM in early pregnancy according to the IADPSG criteria without performing clinical interventions until mid-pregnancy. Women received a $75 \mathrm{~g}$ OGTT by 20 weeks of gestation and again during 24-28 weeks of gestation-the IADPSG-recommended testing period. We diagnosed only those women who exhibited a GDM pattern at this time as having true GDM, and treated them as patients with GDM accordingly. We found that if early-onset GDM was left untreated and simply monitored, approximately half of all patients did not exhibit a GDM pattern with their second OGTT during mid-pregnancy. Such women with apparent GDM in early pregnancy but apparent normal glucose tolerance (NGT) in midpregnancy were classified as "false-positive early GDM." [10]

However, "false-positive early GDM" is a new concept. Moreover, this study is the first to propose this concept, and it remains unclear whether women exhibiting a false-positive early GDM pattern should be considered as having impaired glucose tolerance. Thus, in the present study, we compared the pregnancy outcomes of women with false-positive early GDM to those of women with NGT to investigate whether false-positive early GDM women can indeed be managed in the same way as NGT women.

\section{Methods}

Study design

One hundred-seventeen pregnant women with false-positive early GDM were selected from the TTIGDM study, and 1774 pregnant women with NGT were selected among pregnant women who delivered neonates after 22 weeks of gestation. These populations were then compared. The protocol for this research project has been approved by a suitably constituted Ethics Committee of the institution and it conforms to the provisions of the Declaration of Helsinki. Committee of the Yokohama City University Medical Center, Approval No. F211000053.

\section{Participants}

The participants of this study consisted of 117 individuals selected from the TTIGDM study with falsepositive early GDM, as defined below, who delivered single neonates after 22 weeks of gestation at the Yokohama City University Medical Center. The TTIGDM study was a prospective cohort trial conducted between January 2018 and December 2019 at five secondary and tertiary medical facilities in Japan. Among patients with high-risk GDM, we targeted pregnant women who underwent a $75 \mathrm{~g}$ OGTT prior to 20 weeks of gestation and diagnosed women with GDM if they exceeded IADPSG thresholds defined by one or more of the following: FPG level $\geq 92 \mathrm{mg} / \mathrm{dL}, 1 \mathrm{~h}$ post-prandial glucose $\geq 180 \mathrm{mg} / \mathrm{dL}$, and/or $2 \mathrm{~h}$ post-prandial glucose $\geq 153 \mathrm{mg} / \mathrm{dL}$. The definition of high-risk GDM has previously been described in detail. [10] 
As shown in Figure 1, the enrolled study participants did not receive any therapeutic intervention until the repetition of the $75 \mathrm{~g} \mathrm{OGTT} \mathrm{at} \mathrm{24-28} \mathrm{weeks} \mathrm{of} \mathrm{gestation.} \mathrm{Pregnant} \mathrm{women} \mathrm{who} \mathrm{met} \mathrm{the} \mathrm{IADPSG} \mathrm{criteria}$ at 24-28 weeks gestation were designated as true GDM patients and initiated therapy. Women diagnosed with GDM in early pregnancy who did not meet the diagnostic criteria during the second OGTT were defined as having false-positive early GDM. Women with false-positive early GDM did not receive any therapeutic intervention during gestation.

\section{Control}

The control group comprised 1774 pregnant women diagnosed with NGT by a two-step screening process performed during early and mid-pregnancy and who delivered single neonates after 22 weeks of gestation between January 2018 and December 2019 at the Yokohama City University Medical Center. For the two-step screening process, a $75 \mathrm{~g}$ OGTT was performed, with cutoff values of $95 \mathrm{mg} / \mathrm{dL} \mathrm{during}$ early pregnancy or $140 \mathrm{mg} / \mathrm{dL}$ after $50 \mathrm{~g}$ glucose challenge test (GCT) during mid-pregnancy.

\section{Study outcomes}

We examined maternal characteristics, such as maternal age, pre-pregnancy BMI, and nulliparity rate (\%). Pregnancy outcomes included gestational age (weeks), cesarean delivery rate, emergency cesarean delivery rate, gestational weight gain (GWG), birth weight (g), large for gestational age (LGA) rate, small for gestational age (SGA) rate, and rates of hypertensive disorders of pregnancy (HDP). We examined pregnancy and neonatal outcomes, such as the neonatal intensive care unit (NICU) admission rate, neonatal hypoglycemia, respiratory distress syndrome (RDS), and neonatal hyperbilirubinemia, using both univariate and multivariate analyses.

The amount of GWG was defined as the difference between the body weight at delivery and prepregnancy body weight. LGA neonates were defined as those with a birth weight greater than or equal to the 90th percentile. On the other hand, SGA neonates were defined as those with birth weights below the 10th percentile. Neonatal hypoglycemia was defined as a blood glucose level of $<40 \mathrm{mg} / \mathrm{dL}$. In the falsepositive early GDM group, blood glucose level was measured in all neonates after birth. In the NGT group, neonatal blood glucose level was measured only if neonates were SGA or LGA, if NICU admission was required, or if judged necessary by the neonatologist. The presence or absence of hyperbilirubinemia, defined as the need for phototherapy in neonates, was confirmed in all patients of both groups.

RDS was defined by characteristic findings on chest radiographic examination and oxygen requirement within $24 \mathrm{~h}$ after birth. Macrosomia was defined as a birth weight exceeding $4000 \mathrm{~g}$. Shoulder dystocia was defined clinically as the presence of difficulty in delivering the shoulder after delivery of the neonate's head and the necessity for some form of treatment or procedure.

Statistical analyses

JMP pro 15 (SAS Institute Inc., North Carolina, USA) was used for statistical analyses. The medians and proportions were compared using the Mann-Whitney U-test and Fisher's exact test, respectively. The level 
of statistical significance (p) was set at $<0.05$. In multivariate analyses, the adjusted regression coefficient (aRC), adjusted odds ratio (aOR), and 95\% confidence intervals (Cls) were used.

\section{Results}

Maternal characteristics are shown in Table 1. The median maternal age in the false-positive early GDM group was significantly higher than that of the NGT group (37 vs. 33 years, $p<0.001$ ). Pre-pregnancy BMI was also significantly higher in the false-positive early GDM group than in the NGT group $\left(21.9 \mathrm{~kg} / \mathrm{m}^{2} \mathrm{vs}\right.$. $\left.20.5 \mathrm{~kg} / \mathrm{m}^{2} ; \mathrm{p}<0.001\right)$.

Pregnancy outcomes are shown in Table 2. No significant differences between groups were observed as regards gestational age or cesarean delivery rate.

The false-positive early GDM group had significantly less GWG than the NGT group (8.7 kg vs. $10.3 \mathrm{~kg}$ ). The same result was obtained using multivariate analysis $(\mathrm{p}<0.001, \mathrm{aRC}-0.51,95 \% \mathrm{Cl}[-0.919$ to $-0.116])$. No significant difference was observed in the LGA rate between the NGT (9\%) and false-positive early GDM groups (6.8\%) via either univariate or multivariate analysis $(p=0.43, a O R=.248,95 \% \mathrm{Cl}$ [0.299-1.366]).

Macrosomia and shoulder dystocia did not occur in the false-positive early GDM group. No significant differences were observed between the groups in terms of NICU admission, RDS, or neonatal hyperbilirubinemia. However, neonatal hypoglycemia was more common in the false-positive early GDM group $(p=0.002$, aOR $7.453,95 \% \mathrm{Cl}[2.233-24.875])$.

\section{Discussion}

No significant differences in pregnancy or neonatal outcomes (except neonatal hypoglycemia) were observed between the false-positive early GDM group and the NGT group.

There is no consensus on the screening and intervention for GDM in early pregnancy [11-13], and a systematic review of early-onset GDM also stated that any benefit or possible harm caused by the treatment of early-onset GDM should be investigated as soon as possible. [7] In recent years, several randomized controlled trials (RCTs) on impaired glucose tolerance in early pregnancy have been reported. Harper et al. [14] selected obese women, who are at high risk of GDM, and used a two-step screening method involving a $50 \mathrm{~g} \mathrm{GCT}$ and a $100 \mathrm{~g}$ OGTT to conduct an RCT, wherein patients were divided into either a group, in which GDM screening was carried out until 20 weeks of gestation, and another group, in which the normal GDM screening protocol (at 24-28 weeks of gestation) was carried out. No significant differences in composite primary outcomes, including macrosomia, cesarean delivery, HDP, hyperbilirubinemia, shoulder dystocia, and neonatal hypoglycemia, were observed between the group screened early during pregnancy and the group screened at 24-28 weeks of gestation. Even after limiting their analysis to only those cases diagnosed with GDM, no significant differences were found in 
composite primary outcomes between groups, and it was concluded that screening for GDM in early pregnancy did not improve pregnancy outcomes even in obese women.

Roeder et al. [15] carried out an RCT including pregnant women who exhibited early pregnancy hyperglycemia, defined as high fasting blood glucose or an $\mathrm{HbA} 1 \mathrm{c}$ of $5.7 \%$ or higher in early pregnancy. They examined differences in outcomes between a group that received first trimester therapeutic interventions and a group that received third trimester therapeutic interventions., No significant differences were observed in the rates of macrosomia or of use of pharmacotherapies for blood glucose control between the groups. Although this RCT was underpowered, it indicated that early therapeutic interventions produced no discernible effects even among pregnant women with hyperglycemia in early pregnancy.

Liu et al. [16] limited themselves to low-risk pregnant women in a prospective cohort study in which a 75 g OGTT was performed twice during pregnancy. No significant differences, whether by univariate or multivariate analyses, were noted in LGA, macrosomia, neonatal hyperinsulinemia, neonatal hypoglycemia, or pregnancy-induced hypertension in women who exhibited a GDM pattern only in early pregnancy compared to women with NGT during both early and mid-pregnancy. Our study was similar to that of Liu et al., which was limited to low-risk pregnant women, except for our finding that neonatal hypoglycemia was more common in the false-positive early GDM group. Our findings suggest that a $75 \mathrm{~g}$ OGTT conducted in early pregnancy without any follow-up therapeutic interventions would not significantly affect pregnancy outcomes, even in women with risk factors for GDM.

In contrast, we observed that neonatal hypoglycemia was significantly higher in the false-positive early GDM group. It has been reported that even in healthy term infants, transient hypoglycemia of $<47 \mathrm{mg} / \mathrm{dl}$ is observed in approximately $40 \%$ of cases when blood glucose level monitoring is continued for 5 days after birth.[17] In our control (NGT) group, blood glucose was measured only in cases wherein the neonate was judged to be at risk of neonatal hypoglycemia. Therefore, we presume that some newborn babies were hypoglycemic but were overlooked due to a lack of symptoms. As a result, this may have led to a higher incidence of neonatal hypoglycemia in the false-positive early GDM group, wherein examinations were carried out in all cases.

Our study has several limitations. First, the results may not be applicable to other races, since all participants were Japanese. Second, the number of cases was small, and it is possible that a $\beta$ error occurred. Third, since the subjects of the false-positive early GDM group were aware that they would normally have been diagnosed with GDM in Japan, it is possible that this knowledge caused some lifestyle-related changes. In fact, the false-positive early GDM group had significantly less GWG than the NGT group. The possibility that this may have affected pregnancy outcomes cannot be ruled out. Contrarily, to the best of our knowledge, our study is the first to demonstrate that pregnancy outcomes do not significantly differ between false-positive early GDM pregnant women selected from women with risk factors for GDM and NGT women. 


\section{Conclusion}

In conclusion, pregnant women with false-positive early GDM can be managed similarly to patients with NGT. Moreover, there little clinical significance to patients with GDM exhibiting GDM patterns only during early pregnancy. Therefore, the WHO diagnostic guidelines, which apply the IADPSG criteria during early pregnancy, should be revised.

\section{Abbreviations}

GDM: gestational diabetes mellitus; NGT: normal glucose tolerance; OGTT: oral glucose tolerance test; IADPSG: the International Association of Diabetes and Pregnancy Study Groups; FPG: fasting plasma glucose; $1 \mathrm{hPG}$ : $1 \mathrm{~h}$ postprandial plasma glucose; $2 \mathrm{hPG}: 2 \mathrm{~h}$ postprandial plasma glucose; TTIGDM study: Timing of Therapeutic Intervention for Gestational Diabetes Mellitus study; GCT: glucose challenge test; GWG: gestational weight gain; LGA: large for gestational age; SGA: small for gestational age; HDP: hypertensive disorders of pregnancy; NICU: neonatal intensive care unit; RDS: respiratory distress syndrome; aRC: the adjusted regression coefficient; aOR: adjusted odds ratio; Cls: confidence intervals; RCT: randomized controlled trial

\section{Declarations}

Ethics approval: The protocol for this research project has been approved by a suitably constituted Ethics Committee of the institution and it conforms to the provisions of the Declaration of Helsinki. Committee of the Yokohama City University Medical Center, Approval No. F211000053.

Consent for publication: Not applicable.

Availability of data and materials; The datasets used and/or analysed during the current study are available from the corresponding author on reasonable request.

Competing interests: The authors have no conflicts of interest to declare.

Funding: The research was partially supported by JSPS KAKENHI (grant number JP1919K18675).

Author's contributions: S.N. researched data and wrote the manuscript. S.A. and J.K. contributed to study design and wrote the manuscript. R.S. and S.O. researched data. E.M. finalized the manuscript.

Acknowledgements: Not applicable.

\section{References}

1. Crowther CA, Hiller JE, Moss JR, McPhee AJ, Jeffries WS, Robinson JS. Effect of treatment of gestational diabetes mellitus on pregnancy outcomes. N Engl J Med. 2005; 352: 2477-86. 
2. Landon MB, Spong CY, Thom E, Carpenter MW, Ramin SM, Casey B, et al. A multicenter, randomized trial of treatment for mild gestational diabetes. N Engl J Med. 2009; 361: 1339-48.

3. Metzger BE, Gabbe SG, Persson B, Buchanan TA, Catalano PA, Damm P, et al. International association of diabetes and pregnancy study groups recommendations on the diagnosis and classification of hyperglycemia in pregnancy. Diabetes Care. 2010; 33: 676-82.

4. Mclntyre HD, Sacks DA, Barbour LA, Feig DS, Catalano PM, Damm P, et al. Issues With the Diagnosis and Classification of Hyperglycemia in Early Pregnancy. Diabetes Care. 2016; 39: 53-4.

5. Diagnostic criteria and classification of hyperglycaemia first detected in pregnancy: A World Health Organization Guideline. Diabetes research and clinical practice. 2014; 103: 341-63.

6. Committee of the Japan Diabetes Society on the Diagnostic Criteria of Diabetes Mellitus; Seino Y, Nanjo K, Tajima N, Kadowaki T, Kashiwagi A, Araki E, et al. Report of the committee on the classification and diagnostic criteria of diabetes mellitus. J Diabetes Investig. 2010; 1: 212-28

7. Immanuel J, Simmons D. Screening and Treatment for Early-Onset Gestational Diabetes Mellitus: a Systematic Review and Meta-analysis. Curr Diab Rep. 2017; 17: 115.

8. Raets L, Beunen K, Benhalima K. Screening for Gestational Diabetes Mellitus in Early Pregnancy: What Is the Evidence? J Clin Med. 2021; 10: 1257.

9. Caissutti C, Berghella V. Scientific Evidence for Different Options for GDM Screening and Management: Controversies and Review of the Literature. Biomed Res Int. 2017; 2017: 2746471.

10. Nakanishi S, Aoki S, Kasai J, Shindo R, Obata S, Hasegawa Y, et al. High probability of false-positive gestational diabetes mellitus diagnosis during early pregnancy. BMJ Open Diabetes Res Care. 2020;8.

11. Hagiwara Y, Kasai J, Nakanishi S, Saigusa Y, Miyagi E, Aoki S. Should the IADPSG criteria be applied when diagnosing early-onset gestational diabetes? Diabetes Res Clin Pract. 2018; 140: 154-61.

12. Sweeting AN, Ross GP, Hyett J, Molyneaux L, Constantino M, Harding AJ, et al. Gestational Diabetes Mellitus in Early Pregnancy: Evidence for Poor Pregnancy Outcomes Despite Treatment. Diabetes Care. 2016; 39: 75-81.

13. Hong WY, Biggio JR, Tita A, Harper LM. Impact of Early Screening for Gestational Diabetes on Perinatal Outcomes in High-Risk Women. Am J Perinatol. 2016; 33: 758-64.

14. Harper LM, Jauk V, Longo S, Biggio JR, Szychowski JM, Tita AT. Early gestational diabetes screening in obese women: a randomized controlled trial. Am J Obstet Gynecol. 2020; 222: 495.e1-.e8.

15. Roeder HA, Moore TR, Wolfson MT, Gamst AC, Ramos GA. Treating hyperglycemia in early pregnancy: a randomized controlled trial. Am J Obstet Gynecol MFM. 2019; 1: 33-41.

16. Liu B, Cai J, Xu Y, Long Y, Deng L, Lin S, et al. Early Diagnosed Gestational Diabetes Mellitus Is Associated With Adverse Pregnancy Outcomes: A Prospective Cohort Study. J Clin Endocrinol Metab. 2020; 105.

17. Harris DL, Weston PJ, Gamble GD, Harding JE. Glucose Profiles in Healthy Term Infants in the First 5 Days: The Glucose in Well Babies (GLOW) Study. J Pediatr. 2020; 223: 34-41. e4. 


\section{Tables}

Table 1. Maternal characteristics

\begin{tabular}{|llll|}
\hline & $\begin{array}{l}\text { Normal glucose tolerance group } \\
\mathrm{n}=1774\end{array}$ & $\begin{array}{l}\text { False-positive early GDM } \\
\text { group } \mathrm{n}=117\end{array}$ & $\begin{array}{c}\mathrm{p} \text { - } \\
\text { value }\end{array}$ \\
\hline Age (years) & $33[29-36]$ & $37[34-39]$ & $<0.001$ \\
\hline $\begin{array}{l}\text { Pre-pregnancy BMI } \\
\left(\mathrm{kg} / \mathrm{m}^{2}\right)\end{array}$ & $20.5[19.1-22.6]$ & $21.9[19.5-24.8]$ & $<0.001$ \\
\hline Primipara & $898(51 \%)$ & $57(49 \%)$ & 0.69 \\
\hline Abbreviations: GDM, gestational diabetes mellitus; BMI, body mass index & \\
\hline
\end{tabular}

Table 2. Pregnancy and neonatal outcomes 


\begin{tabular}{|c|c|c|c|c|c|}
\hline & $\begin{array}{l}\text { Normal glucose } \\
\text { tolerance group } \\
\mathrm{n}=1774\end{array}$ & $\begin{array}{l}\text { False-positive early } \\
\text { GDM group } n=117\end{array}$ & $\begin{array}{l}\mathrm{p}- \\
\text { value }\end{array}$ & $\mathrm{aRC}$ & $95 \% \mathrm{Cl}$ \\
\hline $\begin{array}{l}\text { Gestational } \\
\text { weight gain (kg) }\end{array}$ & 10.3 [ 7.7-12.7] & $8.7[5.0-12.0]$ & $<0.001$ & -0.51 & $\begin{array}{l}-0.919- \\
-0.116\end{array}$ \\
\hline $\begin{array}{l}\text { Gestational age } \\
\text { (weeks) }\end{array}$ & $39.2[38.2-40.1]$ & $39.2[38.2-40.1]$ & 0.60 & 0.256 & $\begin{array}{l}0.0158- \\
0.498\end{array}$ \\
\hline \multirow[t]{2}{*}{ Birth weight (g) } & 2982 [ 2700-3233] & 3076 [ 2793-3284] & 0.10 & 46.59 & $\begin{array}{l}-5.050- \\
98.226\end{array}$ \\
\hline & & & $\begin{array}{l}\mathrm{p}- \\
\text { value }\end{array}$ & $\mathrm{aOR}$ & $95 \% \mathrm{Cl}$ \\
\hline LGA & $159(9.0 \%)$ & $8(6.8 \%)$ & 0.43 & 0.248 & $\begin{array}{l}0.299- \\
1.366\end{array}$ \\
\hline SGA & 168 (9.5\%) & $4(3.4 \%)$ & 0.067 & 0.442 & $\begin{array}{l}0.177- \\
1.109\end{array}$ \\
\hline Macrosomia & $11(0.62 \%)$ & $0(0 \%)$ & & & \\
\hline Shoulder dystocia & $13(0.73 \%)$ & $0(0 \%)$ & & & \\
\hline HDP & $140(7.9 \%)$ & $19(16 \%)$ & 0.005 & 1.34 & $\begin{array}{l}0.767- \\
2.358\end{array}$ \\
\hline Cesarean delivery & $470(26 \%)$ & $42(36 \%)$ & 0.027 & 1.024 & $\begin{array}{l}0.679- \\
1.544\end{array}$ \\
\hline $\begin{array}{l}\text { Emergency } \\
\text { cesarean delivery }\end{array}$ & $221(13 \%)$ & $18(15 \%)$ & 0.36 & 0.933 & $\begin{array}{l}0.545- \\
1.599\end{array}$ \\
\hline NICU admission & $296(17 \%)$ & $19(16 \%)$ & 0.84 & 0.848 & $\begin{array}{l}0.504- \\
1.428\end{array}$ \\
\hline $\begin{array}{l}\text { Neonatal } \\
\text { hypoglycemiat }\end{array}$ & $10(0.5 \%)$ & $5(4.3 \%)$ & 0.002 & 7.453 & $\begin{array}{l}2.233- \\
24.875\end{array}$ \\
\hline RDS & $22(1.2 \%)$ & $1(0.9 \%)$ & 0.71 & 0.569 & $\begin{array}{l}0.073- \\
4.421\end{array}$ \\
\hline $\begin{array}{l}\text { Neonatal } \\
\text { hyperbilirubinemia }\end{array}$ & $174(9.8 \%)$ & $13(11 \%)$ & 0.65 & 1.058 & $\begin{array}{l}0.572- \\
1.960\end{array}$ \\
\hline \multicolumn{6}{|c|}{$\begin{array}{l}\text { Abbreviations: GDM, gestational diabetes mellitus; LGA, large for gestational age; SGA, small for } \\
\text { gestational age; HDP, hypertensive disorder of pregnancy; NICU, neonatal intensive care unit; RDS, } \\
\text { respiratory distress syndrome; OR, Odds ratio; RC, Regression Coefficient }\end{array}$} \\
\hline \multicolumn{6}{|c|}{$\begin{array}{l}\text { Values are expressed as median [IQR] or n (\%). Moreover, aOR and aRC have been adjusted for age } \\
\text { and pre-pregnant BMI. } \\
\text { tIn the false-positive early GDM group, blood glucose level was measured in all neonates after birth. } \\
\text { In the NGT group, blood glucose level of neonates was only measured if neonates were SGA or LGA, if } \\
\text { NICU admission was required, or if judged necessary by the neonatologist. }\end{array}$} \\
\hline
\end{tabular}


Figures

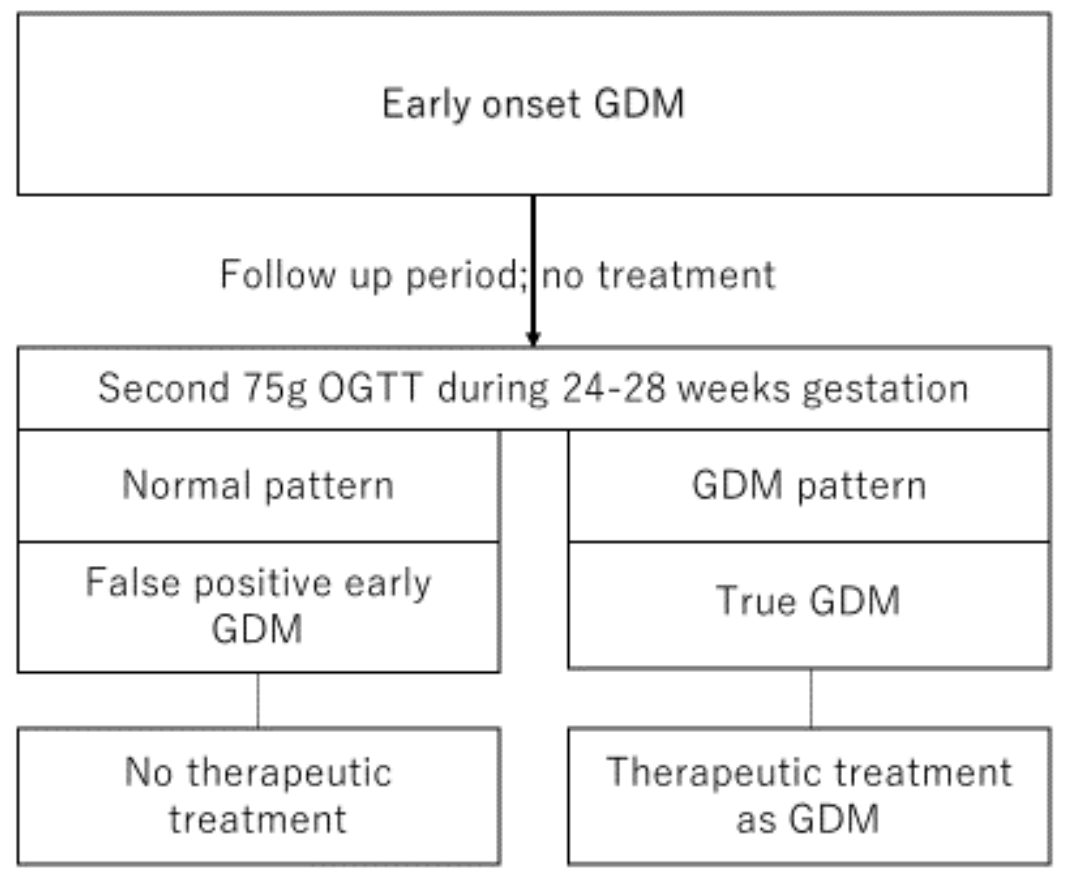

\section{Figure 1}

Timing of Therapeutic Intervention for Gestational Diabetes Mellitus study design

Abbreviations: GDM, gestational diabetes mellitus; OGTT, oral glucose tolerance test 